\title{
Manejo de anestesia total endovenosa en un paciente prematuro con displasia broncopulmonar: Un reporte de caso
}

\author{
Case report: TIVA for premature patient with \\ bronchopulmonary dysplasia
}

Sebastián Amaya ${ }^{1}$, Carlos Quintero MD. ${ }^{2}$, Daniel Ruiz MD. ${ }^{3}$, Héctor S. Cervera ${ }^{1}$, José J. Maya ${ }^{1}$

\begin{abstract}
Patients with hyaline membrane disease (HMD) and bronchopulmonary dysplasia (BPD) can prove to be a challenge when it comes to proper ventilation and perfusion during surgical procedures. We present the case of a 3 month old male infant born at 27 weeks of gestation via caesarean section secondary to premature rupture of membranes which led to eventual neonatal sepsis that was treated appropriately. The patient presents complications related to the preterm birth such as HMD, BPD and an aggressive preterm retinopathy which required urgent vitrectomy to preserve visual function. The surgical procedure was done under total intravenous anesthesia (TIVA) due to the fact that the patient required high frequency ventilation which was not a setting readily available on anesthetic machines. In this case we highlight the importance of knowing the alternatives to inhaled anesthesia in patients with pulmonary disease in which administration of these agents can be erratic and unpredictable.

Method: Case report with appropriate patient consent.
\end{abstract}

\section{RESUMEN}

Los pacientes con enfermedad de membrana hialina (EMH) y displasia broncopulmonar (DBP) representan un desafío al momento de garantizar una adecuada ventilación y perfusión durante procedimientos quirúrgicos. Presen-

\section{Key words:}

Premature,

bronchopulmonary

dysplasia,

total intravenous

anesthesia

\section{Palabras clave:}

Prematuro,

displasia

broncopulmonar, anestesia total

intravenosa

\footnotetext{
Facultad de Medicina, Universidad El Bosque Escuela Colombiana de Medicina, Anesthesiology and Critical Care Interest Group UEB.

2 Médico Anestesiólogo, Hospital Simón Bolivar.

3 Médico Residente de Anestesiología, Posgrado de Anestesiología y Reanimación Universidad El Bosque, Hospital Simón Bolívar.
}

Fecha de recepción: 11 de junio de 2020

Fecha de aceptación: 17 de enero de 2021

\section{ORCID}

0000-0003-1256-2476

\section{Contacto:}

Sebastián Amaya

sebass13@live.com 
tamos el caso de un paciente pediátrico de 3 meses de edad quien nació a las 27 semanas de gestación por cesárea secundaria a una ruptura prematura de membranas que eventualmente llevó a sepsis neonatal, la cual se trató con éxito. El paciente presentó complicaciones propias de la prematurez tales como EMH, DBP, y una retinopatía agresiva que requirió vitrectomía de urgencia para preservar su función visual. El procedimiento quirúrgico se realizó bajo anestesia total endovenosa (TIVA) debido al requerimiento de ventilación oscilatoria de alta frecuencia (VOAF) por el compromiso pulmonar del paciente y la falta de este modo ventilatorio en máquinas anestésicas. En este caso resaltamos la importancia de conocer alternativas a la anestesia inhalatoria en pacientes con enfermedad pulmonar en la que su administración no es posible.

Método: Reporte de caso con consentimiento por la paciente.

\section{Introducción}

a displasia broncopulmonar es una de las principales causas de enfermedad pulmonar crónica en pacientes pediátricos, siendo la mayoría de los casos en prematuros menores a 32 semanas de gestación[1],[2]. La literatura sugiere un manejo anestésico para estos pacientes basado en evitar el óxido nitroso, buenos niveles de oxigenación $\left(\mathrm{PaO}_{2} \approx 50-70 \mathrm{mmHg}\right.$ ), consideración de terapia broncodilatadora perioperatoria, control estricto de la administración de líquidos, entre otras[3]. La anestesia total endovenosa (TIVA) es una alternativa segura en los pacientes pediátricos, reduciendo los riesgos en los que la anestesia inhalatoria puede ser errática e impredecible, además de facilitar algunos procedimientos quirúrgicos, optimizar las condiciones de la cirugía y reducir complicaciones postoperatorias[4]. El uso de esta técnica no es frecuente en pacientes menores de 1 año debido a que no existe un modelo farmacocinético aprobado, sin embargo existen recomendaciones de autores basados en el comportamiento de los medicamentos endovenosos en estos pacientes[4]. Presentamos el caso complejo de un paciente prematuro extremo con bajo peso al nacer de 3 meses de edad, diagnosticado con DBP y retinopatía quien se encontraba en unidad de cuidados intensivos con VOAF e indicación de vitrectomía de urgencias, para la cual se decidió utilizar TIVA dados sus requerimientos ventilatorios. Con este caso queremos destacar que es posible la administración de TIVA en pacientes lactantes con enfermedad pulmonar en los que la vía inhalatoria es poco confiable.

\section{Caso clínico}

Paciente masculino de 37 semanas postconcepcionales con peso actual de $1.755 \mathrm{~g}$ quien nació a las 27 semanas de gestación por cesárea consecuente a ruptura prematura de membranas, llevando a desarrollar sepsis neonatal y endocarditis. Debido a la prematurez extrema, el paciente cursa con retinopatía del prematuro y DBP. El paciente fue llevado a unidad de cuidados neonatales (UCIN) donde presentó cianosis, bradicardia y apnea, por lo que se requirió oxígeno por canula de alto flujo inicialmente. Posteriormente, se solicitó valoración por oftalmología pediátrica, en la cual se determinó la presencia de tortuosidad en los 4 cuadrantes, bilateral, por lo que se consideró que el paciente requería fotocoagulación con láser debido al alto riesgo de presentar desprendimiento de retina. El primer intento de fotocoagulación fue cancelado

\begin{tabular}{lc}
\multicolumn{2}{c}{ Tabla 1. Resultados de exámenes de laboratorio } \\
\hline Laboratorios & Valor \\
Hemoglobina & $9,9 \mathrm{~g} / \mathrm{dL}$ \\
Hematocrito & $30,8 \%$ \\
Plaquetas & $4,87 \times 105 / \mathrm{microliter}$ \\
Calcio & $9,3 \mathrm{mEq} / \mathrm{L}$ \\
Potasio & $4,75 \mathrm{mEq} / \mathrm{L}$ \\
Sodio & $138 \mathrm{mEq} / \mathrm{L}$ \\
$\mathrm{Creatinina}^{2}$ & $0,2 \mathrm{~g} / \mathrm{dL}$ \\
$\mathrm{BUN}^{2}$ & $5 \mathrm{mg} / \mathrm{dL}$ \\
$\mathrm{pH}^{2}$ & 7,27 \\
$\mathrm{pCO}_{2}$ & $56 \mathrm{mmHg}$ \\
$\mathrm{pO}_{2}$ & $39 \mathrm{mmHg}$ \\
$\mathrm{HCO}_{3}$ & $26,6 \mathrm{mEq} / \mathrm{L}$ \\
$\mathrm{Lactato}$ & $5 \mathrm{mmol} / \mathrm{L}$ \\
$\mathrm{Saturación}_{2}$ & $65 \%$ \\
\hline
\end{tabular}

Fuente: Autores. 
por parte del anestesiólogo debido a que presentó broncoespasmo, 2 episodios de apnea y desaturación sin lograr estabilización. En la UCIN presenta aumento del trabajo respiratorio con gases arteriales (Tabla 1) que demuestran un paciente con acidosis mixta e hipoxemia debido al compromiso pulmonar, y se establece el diagnóstico de DBP que requiere finalmente VOAF.

Se realizó una radiografía de tórax la cual evidenció atelectasia masiva en el hemitórax izquierdo y apical derecho y un ecocardiograma en el que se observó un ductus arterioso persistente sin repercusión hemodinámica ni de hipertensión pulmonar (HP). Posteriormente, oftalmología revaloró al paciente evidenciando tracción de la retina y levantamiento periférico por lo que se decidió realizar una vitrectomía anterior de urgencia. Por los antecedentes y exámenes de laboratorio recientes del paciente se clasifica como ASA IV $U$ y se ingresa al quirófano. Por los requerimientos de VOAF en este paciente y los antecedentes pulmonares, se decidió realizar el procedimiento bajo TIVA. Se administró TIVA según el modelo de Steur[5] (Tabla 2) con propofol y remifentanil $0,2 \mathrm{mcg} / \mathrm{kg} / \mathrm{min}$. Durante el procedimiento quirúrgico, el paciente presenta inestabilidad hemodinámica que requiere manejo temprano con dopamina a dosis tituladas de $2,5 \mathrm{mcg} /$ $\mathrm{kg} / \mathrm{min}$, hasta $5 \mathrm{mcg} / \mathrm{kg} / \mathrm{min}$. Debido a la complejidad del procedimiento, el tiempo quirúrgico y la inestabilidad hemodinámica se decidió realizar vitrectomía en dos tiempos siguiendo el mismo esquema anestésico con éxito.

\section{Discusión}

La DBP es una enfermedad pulmonar crónica caracterizada por áreas alternas de fibrosis e hiperinsuflación alveolar, que puede llevar eventualmente a remodelación vascular e HP con daños persistentes en el intercambio gaseoso, funcionalidad de vía aérea, mecánica respiratoria y volúmenes pulmonares que se manifiesta clínicamente de manera variable[6]. Se da principalmente en prematuros como consecuencia de un disbalance entre los mecanismos de lesión y de reparación dados por una interrupción en la etapa tardía del desarrollo pulmonar, que puede llegar a requerir soporte ventilatorio y oxígeno suplementario[6], que a su vez, también contribuyen a una mayor lesión pulmonar[1],[5]. Los criterios diagnósticos y su severidad se establece clínicamente de acuerdo al requerimiento de oxígeno suplementario durante los primeros 28 días de vida ó a las 36 semanas de edad post-concepcional[1]. La intubación

\begin{tabular}{|c|c|}
\hline Tiempo & $\begin{array}{c}\text { Dosis Propofol ( } \mathrm{mg} / \mathrm{kg} / \\
\text { hora) }\end{array}$ \\
\hline 0-10 minutos & 25 \\
\hline 10-20 minutos & 20 \\
\hline 20-30 minutos & 15 \\
\hline 30-40 minutos & 10 \\
\hline 40-100 minutos & 5 \\
\hline Tiempo restante & 2,5 \\
\hline
\end{tabular}

Fuente: Autores agradecen a John Wiley e hijos por el permiso para reproducir el esquema de infusión

endotraqueal y la ventilación mecánica son los principales factores de riesgo para DBP, por lo que utilizar soporte ventilatorio no invasivo parece ser una estrategia útil para evitar la incidencia de la misma y mejorar la supervivencia en bebés prematuros[7]. En las últimas décadas, se han ido desarrollando nuevos modos ventilatorios tratando de disminuir la lesión al parénquima pulmonar, por medio de frecuencias elevadas y pequeños volúmenes como lo hace la VOAF[7]. Este modo de ventilación fue descrito por primera vez en el año 1981 por Marchak y su equipo en un reporte de casos de 8 neonatos con síndrome de distrés respiratorio[8]. En el ambiente perioperatorio, existen situaciones que pueden poner en riesgo al paciente, como lo es la falta de experiencia con la VOAF durante la anestesia quirúrgica, el alto riesgo generado por la ventilación manual requerida durante el transporte de estos pacientes desde la UCI neonatal hacia el quirófano y la no disponibilidad de este modo en las máquinas de anestesia convencionales, lo cual impide el uso de anestésicos inhalados. y requiere del uso de TIVA como alternativa para la administración de la anestesia[7],[8],[9]. Debido a las dificultades mencionadas anteriormente, su uso se ha restringido en situaciones donde se inició la VOAF en UCl y se continúo dentro del quirófano[9]. Sin embargo, se han visto mejoras en el estado respiratorio y el confort durante la cirugía en comparación con ventilación convencional en neonatos resaltando la necesidad desde la anestesiología de familiarizarse con este modo ventilatorio para el cuidado de neonatos de alto riesgo[10]. En nuestro caso, fue necesario continuar la VOAF que no permitía la administración de agentes inhalados, y por lo tanto se decide utilizar TIVA como técnica anestésica. 
Dentro de las metas anestésicas de los pacientes con DBP, se debe evitar el broncoespasmo, la elevación de la resistencia vascular pulmonar (RVP) y la depresión miocárdica, ya que esto podría llevar al desarrollo de una crisis aguda de HP, falla cardiaca derecha, arritmias, choque cardiogénico y muerte[11]. Es indispensable, por tanto, controlar de manera estricta, los parámetros fisiológicos asociados al aumento de la RVP como la hipercapnia, hipoxemia, hipotermia y acidosis, así como mantener un adecuado plano anestésico y bajas presiones de la vía aérea, ya que todos estos factores están asociados al desarrollo de HP[12]. En este paciente, el manejo con TIVA puede ser beneficioso, no sólo por el modo VOAF y las propiedad broncodilatadores del propofol, sino también por no inhibir la vasoconstricción pulmonar hipóxica, a diferencia de los agentes inhalados, convirtiéndola una técnica alternativa interesante en los pacientes con hipoxemia[11]. Por otro lado, cabe resaltar que los agentes volátiles también están asociados a una mayor depresión miocárdica en una mayor medida que el propofol[12].

Al administrar propofol en pacientes lactantes, hay que tener en cuenta ciertas consideraciones farmacocinéticas dentro de las que se encuentran el aumento del compartimiento central y volumen de distribución debido a un mayor porcentaje de agua corporal total que va disminuyendo a manera que se va envejeciendo. Por lo que en estos pacientes se requiere un bolo y velocidad de infusión inicial más alto, sin embargo, es necesario disminuir rápidamente las dosis de infusión hasta llegar a velocidades de mantenimiento mucho menores que en pacientes mayores, debido a que los pacientes lactantes presentan una disminución del aclaramiento del propofol por su inmadurez hepática[4]. Por estas razones hay una discrepancia entre las velocidades de infusión que se usan en pacientes adultos y en neonatos, lo cual es importante tener presente al llevar a cabo esta técnica según el tipo de paciente. Partiendo de esto, se puede revisar el esquema de infusión utilizado en este caso, planteado por Steur y colegas[5] (Tabla 2.) que se diferencia de esquemas en adultos por lo discutido anteriormente. El remifentanil se usó como agente sinérgico para brindar condiciones quirúrgicas óptimas en dosis usuales basadas en el peso del paciente, ya que sus propiedades farmacocinéticas no difieren en gran medida con respecto al paciente adulto, debido a que la actividad de la colinesterasa plasmática se mantiene similar desde el nacimiento hasta la edad adulta[4].

Es importante también discutir terapias alternativas para el manejo anestésico de estos pacientes, como el fentanilo, la morfina, el midazolam, la ketamina y la dexmedetomidina. Con respecto a los opioides de mayor duración, la morfina y el fentanilo tiene ciertos beneficios, ya que incrementa la sincronía con el ventilador, disminuye las concentraciones de adrenalina y cortisol y no aumenta el riesgo de complicaciones neurológicas como la hemorragia intraventricular (HIV) y leucomalacia periventricular (LMP). Sin embargo, estos opioides están asociados a una serie de efectos adversos indeseables en los pacientes prematuros, como la depresión respiratoria y prolongación de la ventilación mecánica; alteraciones de la motilidad gastrointestinal que no permiten la alimentación enteral completa, aumentan el riesgo del síndrome de tapón de meconio, perforaciones, aumento de presión intraabdominal y enterocolitis necrotizante; así mismo están asociados a inmunosupresión y mayor riesgo de sepsis; y también se han involucrado en neuroapoptosis y alteraciones en el neurodesarrollo normal[13].

En cuanto al uso de midazolam en neonatos, se ha disminuido cada vez más su uso ya que está asociado a un aumento significativo de desarrollar HIV, LMP, hipotensión, disminución del índice de oxigenación cerebral, mioclonías, hiperexcitación y aumento de la mortalidad[13],[14]. La ketamina ha sido asociado con neuroapoptosis y neurotoxicidad en estudios en animales con dosis altas o incluso mayores a las permitidas clínicamente, razón por la cual se ha dejado de estudiar en los paciente neonatos[15],[16], sin embargo, se deberían realizar estudios con dosis subanestésicas en una técnica multimodal.

Una alternativa prometedora es la dexmedetomidina, que a pesar de no estar aprobada su uso pediátrico por la FDA, existe una evidencia creciente de su uso beneficioso en los paciente neonatos que requieren ventilación mecánica, ya que ha demostrado varias ventajas con respecto a los opioides y benzodiacepinas. La dexmedetomidina produce hipnosis y analgesia, sin producir depresión respiratoria, lo que se traduce en un retiro más temprano de la ventilación mecánica; no altera la motilidad gastrointestinal y está asociada a una menor incidencia de delirio y síndrome de abstinencia; así mismo tiene efectos inmunomoduladores al inhibir la liberación de citoquinas proinflamatorias y mantener la activación de neutrófilos y macrófagos lo que disminuye el riesgo de lesión pulmonar asociada al ventilador y de sepsis. Además, en los últimos estudios en animales proponen a la dexmedetomidina como un agente neuroprotector, especialmente en cerebros hipóxicoisquémicos, delimitando la lesión cerebral al inhibir la neuroapoptosis[13]. 


\section{Conclusión}

Existen situaciones que obligan a conocer alternativas terapéuticas a la anestesia inhalada, como sucedió en nuestro caso. Por esto, es importante saber que existen diferentes fármacos para la administración de TIVA en lactantes y neonatos que requieren procedimientos urgentes. Es necesario disminuir el uso de opioides de larga acción y benzodiacepinas en este grupo de pacientes, por sus múltiples efectos adversos y promover en el futuro los estudios sobre anestesia libre de opioides, basados en técnicas multimodales que involucren la dexmedetomidina, ya que ha tenido resultados prometedores en los pacientes menores y ketamina a dosis sub-anestésicas.

\section{Referencias}

1. Voynow JA. "New" bronchopulmonary dysplasia and chronic lung disease. Paediatr Respir Rev. 2017;24:17-8.10. https://doi. org/10.1016/j.prrv.2017.06.006

2. Davidson LM, Berkelhamer SK. Bronchopulmonary Dysplasia: Chronic Lung Disease of Infancy and Long-Term Pulmonary Outcomes. J Clin Med.2017;6(1):4. https://doi. org/10.3390/jcm6010004

3. Almedia-Chen GM. Handbook of Pediatric Anesthesia. 1st ed. New York (USA): McGraw-Hill Education; c2015. Chapter 20, Bronchopulmonary Dysplasia; p.80-83.

4. Gaynor J, Ansermino JM. Paediatric total intravenous anaesthesia. BJA Educ. 2016;16(11):36973. https://doi.org/10.1093/ bjaed/mkw019

5. Steur RJ, Perez RS, De Lange JJ. Dosage scheme for propofol in children under 3 years of age. Paediatr Anaesth.2004;14(6):462-467. https://doi.org/10.1111/j.14609592.2004.01238.x

6. Thekkeveedu R, Guaman MC, Shivanna B. Bronchopulmonary dysplasia: A Review of Pathogenesis and Pathophysiology. Respir Med. 2017; 132:170-
177. https://doi.org/10.1016/j. rmed.2017.10.014

7. Jain D, Bancalari E. New Developments in Respiratory Support for Preterm Infants. Am J Perinatol. 2019;36(S 02):S13-17. https://doi. org/10.1055/s-0039-1691817

8. Marchak BE, Thompson WK, Duffty $P$, et al. Treatment of RDS by high-frequency oscillatory ventilation: a preliminary report. J Pediatr. 1981;99(2):287-292. https://doi.org/10.1016/S00223476(81)80480-5

9. Pelosi P, Brusasco C, Abreu MG de. Principles and Practice of Mechanical Ventilation. 3rd ed. New York (USA): The McGraw-Hill En: Tobin MJ, editor. Principles and Practice of Mechanical Ventilation. 3.a ed. New York, NY: The McGraw-Hill Companies; c2013. Chapter 24, Mechanical Ventilation During General Anesthesia.

10. Bouchut JC, Godard J, Claris O. High-frequency Oscillatory Ventilation. Anesthesiology. 2004; 100(4): 1007-1012. Disponible en: https://anesthesiology.pubs.asahq.org/article. aspx?articleid=1943214. https:// doi.org/10.1097/00000542200404000-00035

11. Orphananesthesia.eu [internet]. Germany: Bronchopulmonary Dysplasia; c2016 [cited 2020 Jul
01]. Available at: https://www. orpha.net/data/patho/Ans/en/ BronchopulmonaryDysplasia_US_ en_ANS_ORPHA70589.pdf

12. Lauer $R$, Vadi M, Mason L. Anaesthetic management of the child with co-existing pulmonary disease. BJA Br J Anaesth. 2012;109(suppl1):47-59. https:// doi.org/10.1093/bja/aes392

13. McPherson C. Sedation and Analgesia in Mechanically Ventilated Preterm Neonates: Continue Standard of Care or Experiment? J Pediatr Pharmacol Ther JPPT. 2012;17(4):351-64. https://doi.org/10.5863/15516776-17.4.351

14. Pacifici GM. Clinical Pharmacology of Midazolam in Neonates and Children: Effect of DiseaseA Review. Donn SM, editor. Int J Pediatr. 18 de febrero de 2014;2014:309342. https://doi. org/10.1155/2014/309342

15. JL Haidon, FRCA, M Cunliffe, MB BS FRCA FFPMRCA, Analgesia for neonates, Continuing Education in Anaesthesia Critical Care \& Pain, Volume 10, Issue 4, August 2010, Pages 123-127. https://doi. org/10.1093/bjaceaccp/mkq016

16. Adnan T. Bhutta, Ketamine: A Controversial Drug for Neonates, Seminars in Perinatology, 2007; 31(5): https://doi.org/10.1053/j. semperi.2007.07.005 